\title{
Determination of Synthetic Impurities and Metabolic Products of F-ADAM, a Positron Emission Tomography Imaging Agent for Serotonin Transporter (SERT) Using HPLC-Tandem Mass Spectrometry*
}

\author{
Wei-Hsi Chen ${ }^{1 \#,}$, Chyng-Yann Shiue ${ }^{2,3}$ \\ ${ }^{1}$ Chemistry Division, Institute of Nuclear Energy Research, Taiwan \\ ${ }^{2}$ PET Center, Department of Nuclear Medicine, National Taiwan University Hospital, Taiwan \\ ${ }^{3}$ PET Center, Department of Nuclear Medicine, Tri-Service General Hospital, Taiwan \\ Email: "whchen@iner.gov.tw
}

How to cite this paper: Chen, W.-H. and Shiue, C.-Y. (2020) Emission Tomography Imaging Agent for Serotonin Transporter (SERT) Using HPLC-Tandem Mass Spectrometry. Pharmacology \& Pharmacy, 11, 282-298.

https://doi.org/10.4236/pp.2020.1110023

Received: September 10, 2020

Accepted: October 27, 2020

Published: October 30, 2020

Copyright $\odot 2020$ by author(s) and Scientific Research Publishing Inc. This work is licensed under the Creative Commons Attribution International License (CC BY 4.0).

http://creativecommons.org/licenses/by/4.0/ (c) (i) Open Access

\begin{abstract}
A high performance liquid chromatography tandem mass spectrometry (HPLC-MS/MS) analytical method was developed to determine the identity of impurities resulting from the synthesis of

$\mathrm{N}, \mathrm{N}$-dimethyl-2-(2-amino-4-fluorophenylthio)benzyl-amine (F-ADAM), as well as its metabolic products by rat liver microsomes. ${ }^{18} \mathrm{~F}-\mathrm{ADAM}$ is an important positive electron emission ligand commonly employed as a radio-imaging agent for serotonin transporter (SERT) in the brain. F-ADAM and its derivatives were separated using HPLC on a C4-phenyl column with an ammonium formate aqueous buffer/acetonitrile programmed mobile phase. Synthetic contaminants and metabolic products were identified using fragmentation spectra obtained by tandem mass spectrometry. We show that F-ADAM is unstable in methanol, and propose the use of acetonitrile to generate optimal chromatogram. A Cl-substituted species was found to be the major impurity resulting from the F-ADAM synthetic process. The metabolic products of F-ADAM by rat liver microsomes were characterized by oxidization of the sulfur moiety to sulfoxide, demethylation of the dimethylamine moiety, and oxidative defluorination/deamination. These results elucidate the by-products of F-ADAM synthetic and metabolic processes, and provide direction for the application of this imaging agent to biosystems properly.
\end{abstract}

${ }^{\star}$ Determination of Impurities and Metabolites of F-ADAM, a PET agent for SERT. 


\section{Keywords}

Liver Microsomes, Metabolites, Impurities, F-ADAM, Serotonin Transporter

\section{Introduction}

Serotonin is a critical neurotransmitter in the central nervous system (CNS) which regulates numerous physiological processes including mood, cognition, learning, memory, sleeping, depression, appetite, and aggressive behavior [1] [2] [3]. Serotonin transporter (SERT) regulates serotonin levels by reuptaking serotonin from the synaptic cleft [4]. Abnormal operation of the serotonin receptor and/or SERT has been linked to various mental disorders [4] [5]. Thus, SERT is the primary target of selective serotonin reuptake inhibitors (SSRIs), a group of drugs widely prescribed for the treatment of neurologic and psychiatric disorders such as depression, anxiety, suicide, schizophrenia, eating disorders, and drug addiction [6] [7] [8]. For the study of neurologic and psychiatric disorders in the context of treatment with SSRIs, non-invasive imaging approaches such as positron emission tomography (PET), and single photon emission computed tomography (SPECT) are powerful tools to guide drug development studies and evaluate SERT functionality [7] [8] [9] [10]. Because these techniques rely on radiolabelled molecular probe, it is critical to develop SPECT or PET-compatible radiotracers with high affinity and specificity for SERT [9].

Several radioligands for SERT imaging with SPECT or PET have been developed in the last two decades with suitable affinity and selectivity for SERT. The most widely studied radioligands are derivatives of

N,N-dimethyl-2-(2-amino-4-methylphenylthio)benzylamine (ADAM) labelled with either ${ }^{123} \mathrm{I}\left({ }^{123} \mathrm{I}-\mathrm{ADAM}\right)$ for SPECT imaging [11] [12], ${ }^{18} \mathrm{~F}\left({ }^{18} \mathrm{~F}-\mathrm{ADAM}\right)$ [3] [7] [9] for PET imaging, or ${ }^{11} \mathrm{C}\left({ }^{11} \mathrm{CH}_{3}-\mathrm{ADAM}\right)$ [8] [13] for PET imaging. Biodistribution studies for ${ }^{123} \mathrm{I}$-ADAM revealed a higher affinity $(\mathrm{Ki}=0.013 \mathrm{nM})$ and greater specificity for SERT ( $>1000$ fold) compared to norepinephrine and dopamine transporters ( $\mathrm{Ki}=669 \pm 80$ and $840 \pm 100 \mathrm{nM}$, respectively) [11].

The radio-isotopically labelled fluoride derivative of ADAM, $4-{ }^{18} \mathrm{~F}-\mathrm{ADAM}$, was introduced in 2003 by Shiue and co-workers [14], and a facile synthetic procedure using a fully automated one-pot two-step method [15]. A series of studies of application of ${ }^{18} \mathrm{~F}$-ADAM to validate the ligand as a SERT imaging agent [7], applied in monkey brain [3], Parkinsonian rat models [16], and for the evaluation of neural transplantation treatment outcomes in Parkinsonian rats [17]. ${ }^{18} \mathrm{~F}$-ADAM PET SERT imaging has also been applied to evaluate brain function recovery in rats with 3,4-methylenedioxy methamphetamine (MDMA) induced behavior disorders treated with resveratrol [10]. Additionally, this group synthesized and compared various ${ }^{18} \mathrm{~F}$-labelled positions on the ADAM parent molecule for SERT binding affinity, showing that $4{ }^{-18} \mathrm{~F}-\mathrm{ADAM}$ was the best candidate for optimized SERT PET imaging [18]. Therefore, $4{ }^{-18} \mathrm{~F}-\mathrm{ADAM}$ 
holds great promise for the evaluation for serotonin-linked disorders.

The metabolic products formed from xenobiotic species when introduced into biosystems are directly related to their safety and efficacy, and must be characterized to ensure minimal toxicity [19]. Furthermore, for radio-labelled imaging agents, the integrity of the molecular probe must be maintained to maximize its contrast and affinity for the target organ. In general, biotransformation of chemical species into smaller or more polar molecules for detoxification and elimination from the body is accomplished by the CYP enzyme family. This process may also result in higher toxicity or, if intentionally exploited, pharmacological efficacy as in the case of prodrugs.

Similarly, the identification of concomitant impurities within drug substances and their unstable deteriorative products is paramount to ensure drug quality and safety [20]. The determination of impurities may also inform improved synthesis, purification, or storage conditions.

In this study, the fragmentation pathway of $4-{ }^{19} \mathrm{~F}-\mathrm{ADAM}$ (non-radioactive version) was elucidated by electrospray ionization triple quadrupole tandem mass spectrometry (ESI QqQ MS/MS). Additionally, an HPLC analytical method was employed to quantify impurities, derivatives, and metabolites of F-ADAM. The identity of synthetic impurities and metabolites resulting from rat liver microsome exposure were determined by tandem mass spectrometry. These results provide a more complete picture regarding the preparation and application of $4{ }^{18}$ F-ADAM.

\section{Materials and Methods}

\subsection{Materials and Reagents}

Analytical-grade chemicals for LC-MS were used as received without further purification. Acetonitrile (HPLC and MS grade), ammonium formate, and dimethyl sulfoxide (DMSO) were all purchased from Merck (Darmstadt, Germany). Ultrapure water (total organic carbon $<5 \mathrm{ppb}$, resistivity $\geq 18.2 \mathrm{M} \Omega-\mathrm{cm}$ at $25^{\circ} \mathrm{C}$ ) was obtained using a Smart DQ3 reverse osmosis reagent water system (Merck Millipore, Billerica, MA, USA) fitted with a $0.22 \mu \mathrm{m}$ polyvinylidene fluoride (PVDF) filter. Rat liver microsomes, solutions of NADPH coenzymes A and B, were all purchased from BD Biosciences (Bedford, MA, USA) and stored at $-70^{\circ} \mathrm{C}$.

The compound of interest, $4-^{19} \mathrm{~F}-\mathrm{ADAM}$, was prepared by Shiue's group (Figure 1). The product was characterized by TLC, melting point, ${ }^{1} \mathrm{H}$ NMR, and elemental analysis [14], and stored at $-20^{\circ} \mathrm{C}$ prior to analysis by HPLC or MS.

\subsection{Analytical Equipment}

The levels of F-ADAM impurities and metabolites were determined using an HPLC system (Agilent 1100/1200 series, Palo Alto, CA, USA) with an online degasser, binary pump, thermostat autosampler $\left(10^{\circ} \mathrm{C}\right)$, temperature-controlled column oven, and diode array detector (DAD, detection wavelength at $240 \mathrm{~nm}$ ). 
Data were acquired and processed using Agilent ChemStation 10.02 software. The HPLC system was coupled with a MS/MS apparatus (4000 QTRAP, AB Sciex, Concord, ON, Canada), operated using Analyst 1.6.2 software. The mass spectrometer was equipped with an electrospray ionization (ESI) source and a triple quadrupole linear ion trap (QqQ LIT) mass detector. The mass analyser was operated in positive-ion detection mode.

\subsection{Analytical Method}

The HPLC analytical method for F-ADAM employed a butyl-phenyl modified silica gel column (length $10 \mathrm{~cm}$, i.d. $3 \mathrm{~mm}$, particle size $3 \mu \mathrm{m}$, Gold Phenyl, Thermo Fisher) with a guard column. The column temperature was set at $23^{\circ} \mathrm{C}$. The mobile phase was delivered by a two-pump programmed gradient (Table 1): pump A: formic acid $1 \mathrm{~mL}$, ammonium formate $0.31 \mathrm{~g}$ dissolved in $1 \mathrm{~L}$ DI water and degassed in ultrasonic bath for $20 \mathrm{~min}$; pump B: acetonitrile $100 \%$. The flow rate was $0.5 \mathrm{~mL} / \mathrm{min}$ and the chromatographic time was $11 \mathrm{~min}$ per injection. The detection wavelength was $240 \mathrm{~nm}$. The sample volume was $5 \mu \mathrm{L}$.

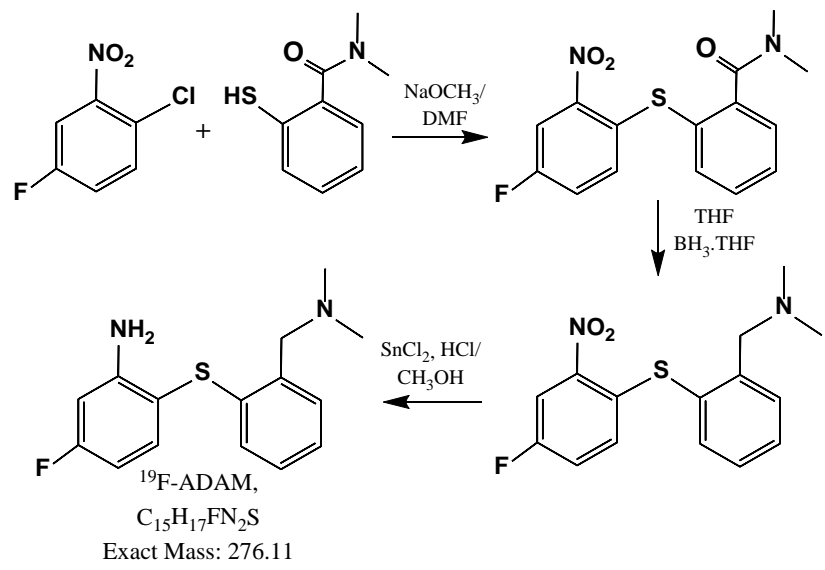

Figure 1. Synthetic route and molecular structure for $4-{ }^{19}$ F-ADAM [14].

Table 1. Gradient program for chromatographic separation of F-ADAM and its derivatives.

\begin{tabular}{cccc}
\hline Time, min & Pump A (\%), aqueous buffer & Pump B (\%) acetonitrile & Flow rate, $\mathrm{mLmin}^{-1}$ \\
\hline 0 & 90 & 10 & 0.5 \\
0.5 & 90 & 10 & 0.5 \\
1 & 80 & 20 & 0.5 \\
6 & 20 & 80 & 0.5 \\
8.5 & 20 & 80 & 0.5 \\
8.55 & 90 & 10 & 0.75 \\
10.55 & 90 & 10 & 0.75 \\
10.6 & 90 & 10 & 0.5 \\
\hline
\end{tabular}




\subsection{Procedures Used for Biotransformation Studies of F-ADAM}

To generate metabolites of F-ADAM using rat liver microsomes, F-ADAM was dissolved in DI water-DMSO (1:1 mixture) as $1000 \mathrm{ppm}$ and processed the solution following the guidelines for using of liver microsome [21] [22]. After periodic incubation $(5,30,60,90$, and $120 \mathrm{~min})$, the reaction was stopped by adding iced acetonitrile ( $1: 1$ volume) and vortexing, and the solutions were centrifuged at $6000 \mathrm{rpm}$ at $4^{\circ} \mathrm{C}$ for $10 \mathrm{~min}$. The supernatant was filtered through a $0.22 \mu \mathrm{m}$ disk membrane (PVDF) into a labelled HPLC vial and stored at $-20^{\circ} \mathrm{C}$.

\section{Results and Discussion}

\subsection{Mass Analysis of F-ADAM and Its Fragmentation Ions}

Prior to identification of impurities/derivatives, mass analysis parameters for F-ADAM were optimized. A solution of 100 ppm F-ADAM in 1:1 methanol/aqueous ammonium formate ( $5 \mathrm{mM})$ (1:1) was injected to the 4000QTRAP by syringe pump at flow rate $10 \mu \mathrm{L} \cdot \mathrm{min}^{-1}$. The intensity was optimized at DP 30 V, curtain gas 10 - 30 psi, IS $5500 \mathrm{~V}$, CE 20 - 30 V, EP 10 - 30 V, and GS1, GS2 both at $10 \mathrm{psi}$ by parameters ramp mode with positive ion monitoring.

The representative molecular weight of F-ADAM is 276 and the measured $m / z$ was 277,278 and 279 with intensity around $16: 3: 1$, in agreement with the protonated F-ADAM molecular weight distribution. The product ion spectra of protonated F-ADAM at $\mathrm{m} / \mathrm{z} 277$ showed fragments at $\mathrm{m} / \mathrm{z} 232,230,217,215$, $212,199,198,185,166,154,137$, and 121 (Figure 2). The proposed fragmentation ion structures are presented in Figure 3. The tandem mass spectra of 4-F-ADAM are comparable to 4-I-ADAM [23]. The dimethylamine group of F-ADAM and I-ADAM is readily dissociated $(\Delta m / z=-45)$. Therefore neutral loss (NL) of $m / z 45\left(\mathrm{HN}\left(\mathrm{CH}_{3}\right)_{2}\right)$ may provide evidence for the identification of unknown F-ADAM derivatives. The product ions with $\mathrm{m} / \mathrm{z}=230,215,212,198$, 121 preferred to rearrange into more stable intermediate structures with $\pi$ - electron delocalization and the dispersed charge facilitated by phenyl and benzyl groups and the lone pair electrons of $\mathrm{N}$ and $\mathrm{S}$, respectively. The structures shown in [23] with mono or bi-radical product ions were less stable and lower probability. The tandem mass spectra and fragmented ion structures indicated that phenyl-amino group did not dissociate from the parent ion $\left(\Delta m / z=-17, \mathrm{NH}_{3}\right.$ than 277) as aliphatic amines. Instead, at the secondary fragmentation stage, ammonia dissociated from $\mathrm{m} / \mathrm{z}=232$ to form $\mathrm{m} / \mathrm{z}=215$ accompanied by molecular rearrangement. The top three ion pairs in terms of intensity were $277>$ 232, $277>230$, and $277>198$ which are available for HPLC coupled with mass spectrometer and multiple reaction monitoring (MRM) quantitation of F-ADAM in complicated matrix solution and excluding of interfering signals.

\subsection{Development of an HPLC Analytical Method for F-ADAM}

The chromatogram of F-ADAM dissolved in methanol revealed that the peak of 
F-ADAM diverged after overnight storage at $10^{\circ} \mathrm{C}$ with retention time $\left(t_{\mathrm{R}}\right)$ at $4.61 \mathrm{~min}$ and $4.88 \mathrm{~min}$, respectively and peak height around 45:55. When the solution was freshly prepared, the resulting peak exhibited a $t_{R}$ at $4.7 \mathrm{~min}$. The mass spectrum of the solution stored overnight showed an $\mathrm{m} / \mathrm{z}$ for the impurity at 289, and the fragmentation spectrum is shown in Figure 4. Based on these results, it is believed that F-ADAM is unstable in methanol due to a methoxyl substitution at the fluoride position. It results from strong electronegativity and good leaving character for fluoride, while the solvent possesses nucleophilic reactivity. Thus, we suggested that F-ADAM should be dissolved in acetonitrile (or aprotic solvents) for the next works.

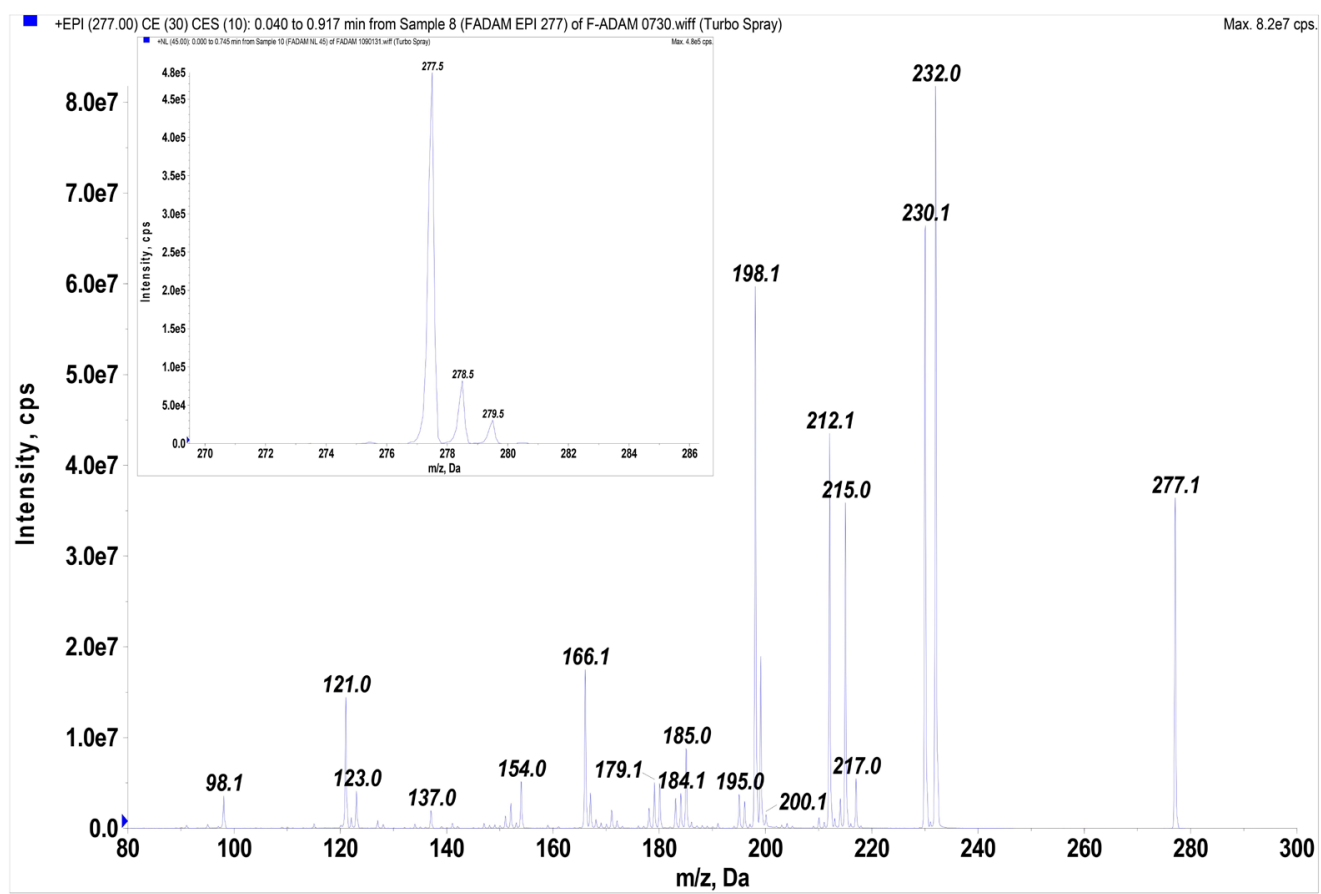

Figure 2. Tandem mass spectra $\left(\mathrm{MS}^{1}\right.$ and $\mathrm{MS}^{2}$ at $\left.m / z=277\right)$ of F-ADAM.<smiles>[CH2]c1ccccc1Sc1ccc(F)cc1N(C)C(C)C(C)N(C)Cc1ccccc1Sc1ccc(F)cc1N</smiles><smiles>Nc1cccc2c1sc1ccccc12</smiles>
$\mathrm{m} / \mathrm{z}: 212$<smiles>[CH2+]c1ccccc1Sc1ccc(F)cc1</smiles>
$\mathrm{m} / \mathrm{z}: 217$<smiles>Fc1ccc2c(c1)[CH]c1ccccc1S2</smiles><smiles>Fc1ccc2sc3ccccc3c2c1</smiles>
m/z: 215<smiles>Fc1ccc2c(c1)N[CH]c1ccccc1Sc1ccc(F)cc1N2</smiles>
$\mathrm{m} / \mathrm{z}: 230$

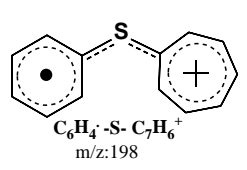<smiles>CN(C)Cc1ccccc1S</smiles><smiles>c1ccc(Sc2ccccc2)cc1</smiles><smiles>F[C+]1[C+]C=CC=C1</smiles><smiles>[C-]#Sc1ccc(F)cc1N</smiles>

Figure 3. Proposed fragmentation structures resulting from the mass analysis of 4-F-ADAM. 


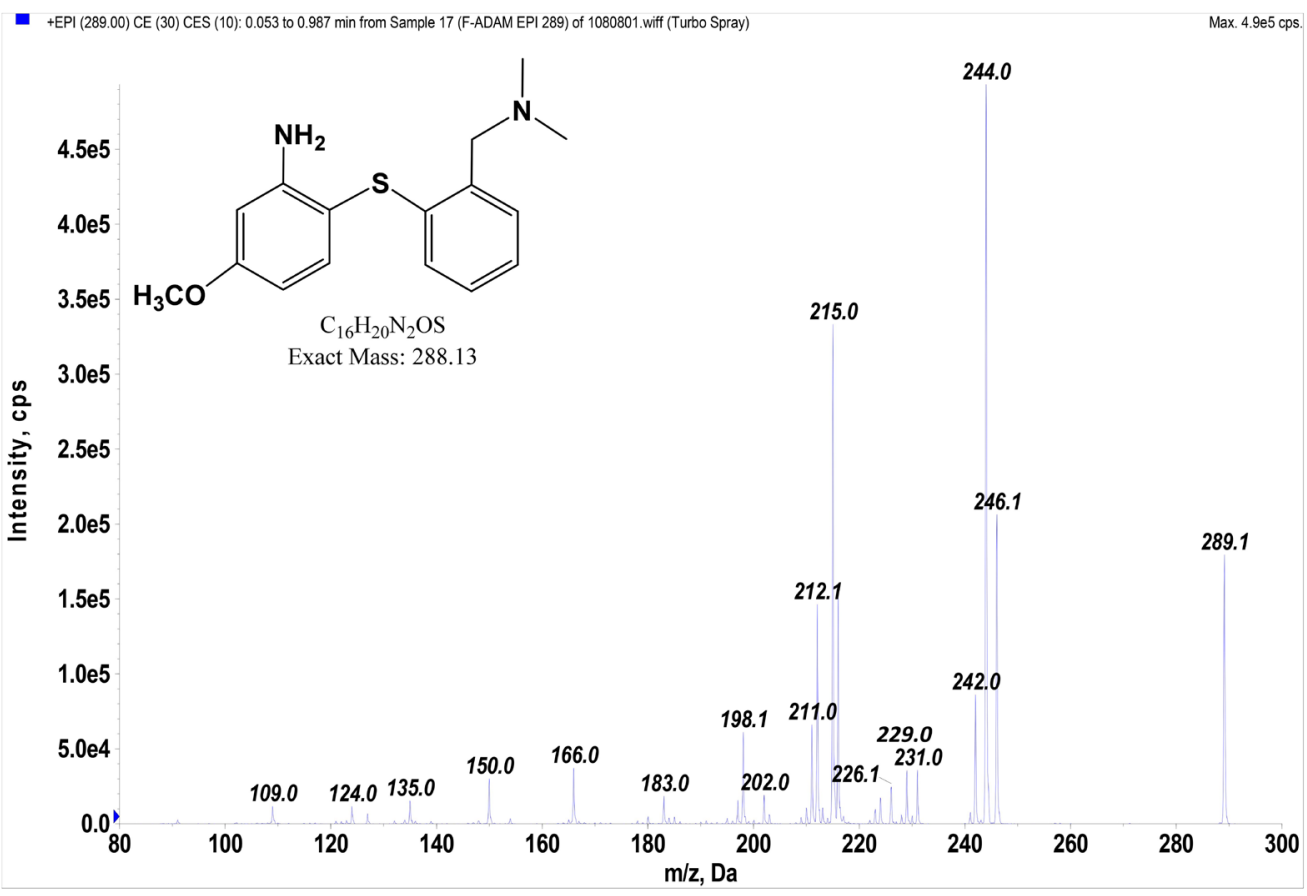

Figure 4. Fragmentation spectra and proposed identity of the methanolic degradation product of F-ADAM when dissolved in methanol.

HPLC of F-ADAM was carried out on a butyl-phenyl modified silica gel column based on the $\pi-\pi$ interaction of the phenyl and benzyl rings contained in the F-ADAM structure with the phenyl-modified column. By comparing the HPLC separation efficiency with methanol or acetonitrile as the pump B solvent, it is clear from the chromatographic results that acetonitrile is a more suitable solvent than methanol. The peak of F-ADAM was broader with methanol compared to acetonitrile (theoretical plate number, N, 10010 vs. 18500). Based on the optimal chromatographic characteristics, the formic acid aqueous buffer/acetonitrile gradient mobile phase coupled with the C4-phenyl column was employed for the study of F-ADAM. A typical chromatogram for F-ADAM is shown in Figure 5 with $t_{R}$ at 5.4 min, a dynamic range between 5 and 375 ppm, a correlation coefficient $(r)>0.9999$ with slope 34.3 area unit per ppm, and an estimated lower limit of quantification (LLOQ) of 1 ppm by UV $(240 \mathrm{~nm})$. The chromatographic purity of F-ADAM was $97.5 \%$.

\subsection{Identification of Impurities within Solutions of F-ADAM}

Several impurities coexisted with F-ADAM post-synthesis. The most significant impurity displayed a $t_{R}$ at 6.01 min with an aboundance of $2.14 \%$. The first order mass spectra $\mathrm{MS}^{1}$ revealed the impurity at $\mathrm{m} / \mathrm{z} 311$ and 313 with an intensity of about 2:1. These data suggested the compound contains a $\mathrm{Cl}$ moiety. Collision induced dissociation (CID) for $\mathrm{m} / \mathrm{z} 311$ and 313 respectively demonstrated that $311 \rightarrow 266,264,249,231,230,214,198,184,166$; and $313 \rightarrow 268,266,251,248$, $232,231,230,214,198,184,166$ (Figure 6). The supposed fragmentation structures shown in Figure 7 indicate that the impurity is a chloro-substituted ana- 
logue. However, the position of the $\mathrm{Cl}$ moiety on the aromatic carbon is unclear. Thus, the structure showed in Figure 7 is one of possibility. An analysis of the synthetic process suggests this impurity is a by-product of starting material, 2-Cl-5-F-nitrobenzene.

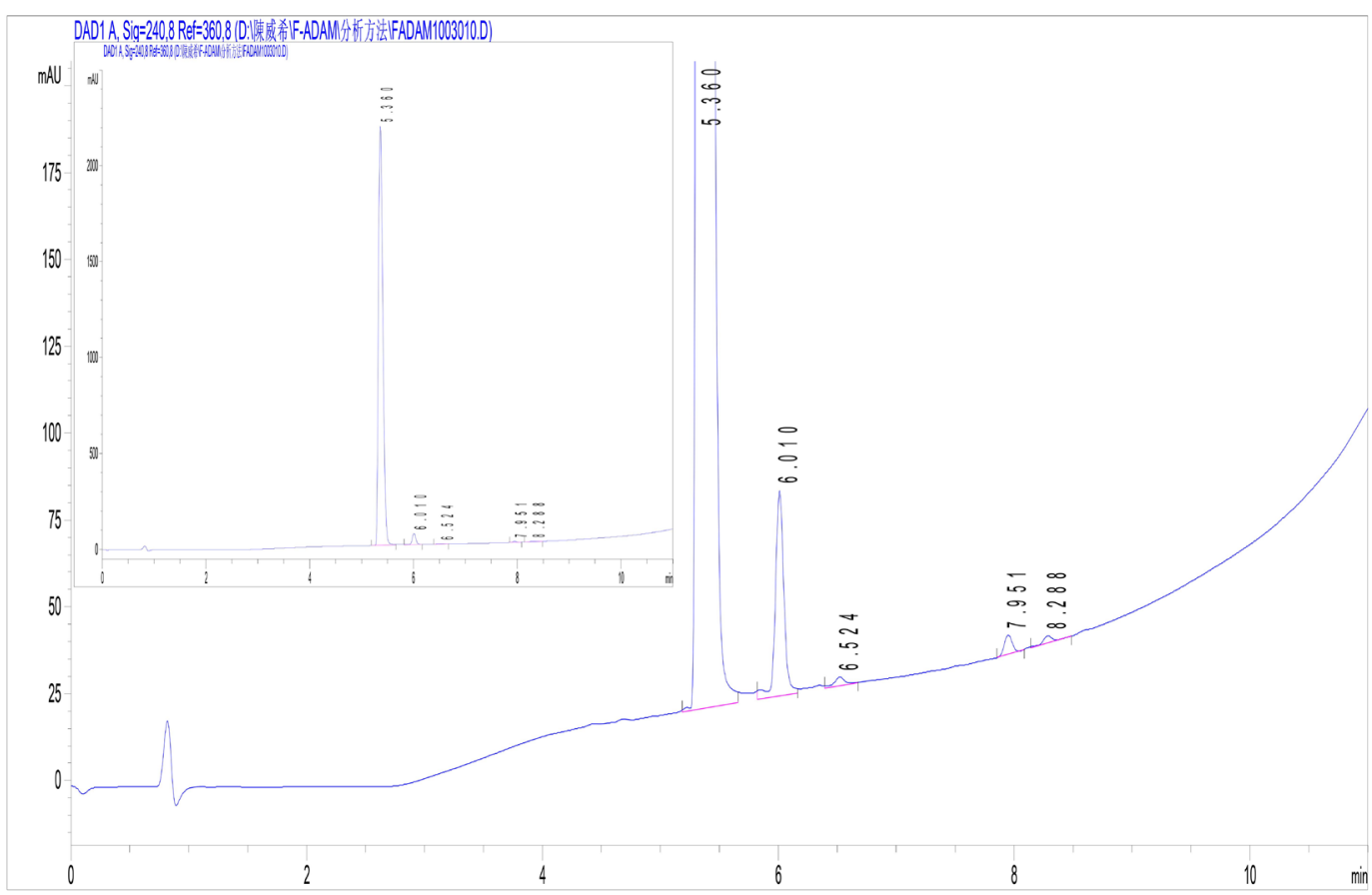

Figure 5. Chromatogram of F-ADAM dissolved in acetonitrile and formic acid aqueous-acetonitrile mobile phase.
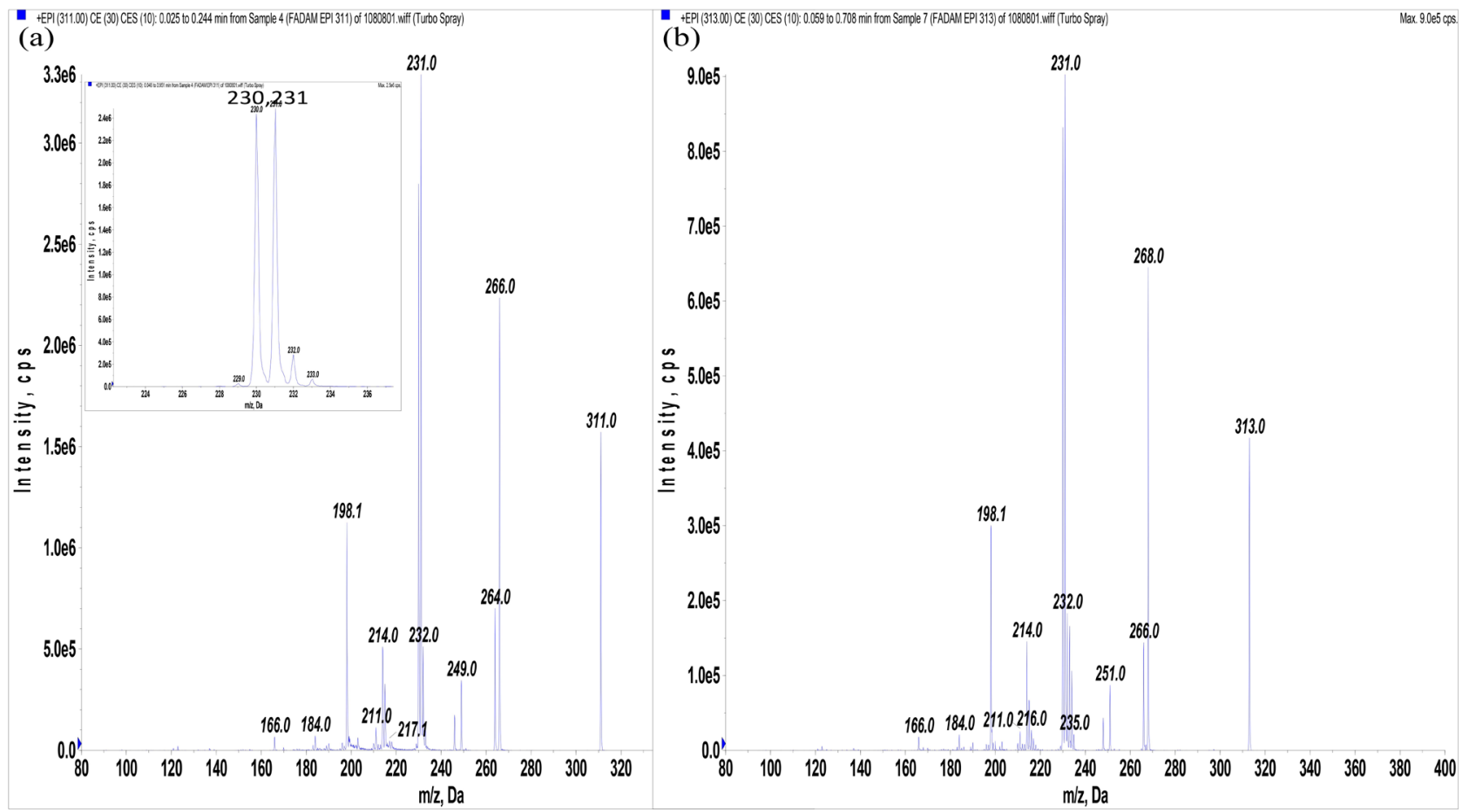

Figure 6. Tandem mass spectra of the major impurity in F-ADAM at $m / z 311$ (a) and 313 (b). 


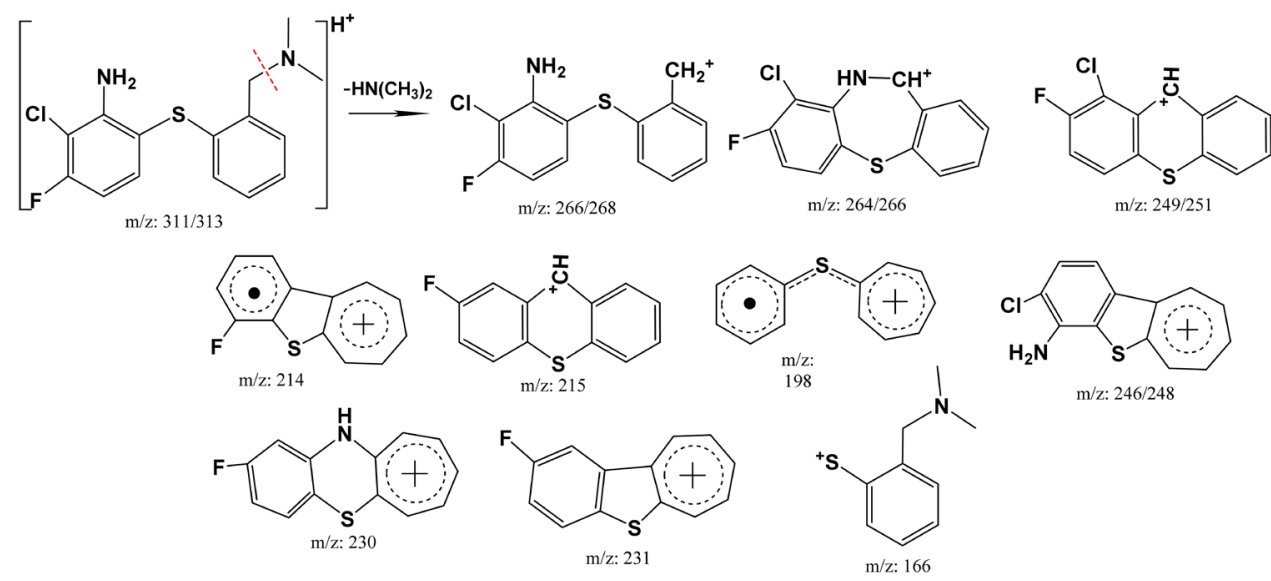

Figure 7. Proposed molecular and fragmented ion structures for $m / z=311$ and 313 resulting from the major impurity in F-ADAM.

The molecular ion $\mathrm{m} / \mathrm{z}$ for other minor impurities (abundance about $0.08 \%$ $0.13 \%)$ identified were probed by $\mathrm{MS}^{1}$ and CID. Their identities were determined based on tandem mass data $\left(\mathrm{MS}^{2}\right)$ and are summarized in Table 2. One of the minor impurities, marked by a nitro-substitution at the amine of F-ADAM, was noted to be an intermediate species in the F-ADAM synthesis route. The levels of both minor impurities were near to LLOQ of the F-ADAM HPLC-UV analytical method.

\subsection{Identification of Metabolites of F-ADAM Generated by Rat Liver Microsomes}

The liver is the major organ responsible for transformation and degeneration of xenobiotics in the body. The fate of pharmaceutical agents in the body including processes such as adsorption, distribution, metabolism, and elimination (ADME) relate to drug safety and function. Importantly, a given molecule's metabolic scheme is related to the distribution and elimination of the drug. For radio-imaging agents, metabolism of the molecular probe impacts imaging quality, contrast, and precision. Liver microsome, which is derived from the membranes of the endoplasmic reticulum of liver cells [21], is commercially available and convenient to use. Microsomes provide an enriched source of membrane bound drug metabolizing enzymes [21]. The methodology for determining the identities of F-ADAM impurities and degradation products was applied to study the metabolic scheme of F-ADAM by rat hepatic enzymes. The chromatogram of F-ADAM incubated with rat liver microsomes for 90 min is showed in Figure 8. There were five metabolite-related peaks observable in the chromatogram (UV detector), of which three were major and two were minor metabolites. The molecular ions $\mathrm{m} / \mathrm{z}$ for six metabolites $\left(\mathrm{MS}^{1}\right)$ in biotransformed solutions of F-ADAM (120 min) revealed signal at 334, 321, 319, 293, 264, and 263, excluding the background and noise signals compared to pre-incubation and free F-ADAM solutions. NL scanning $(\Delta m / z=-45$ and -32$)$ showed that $m / z=293$, 
Table 2. The proposed minor impurities in F-ADAM and their tandem mass spectra data.
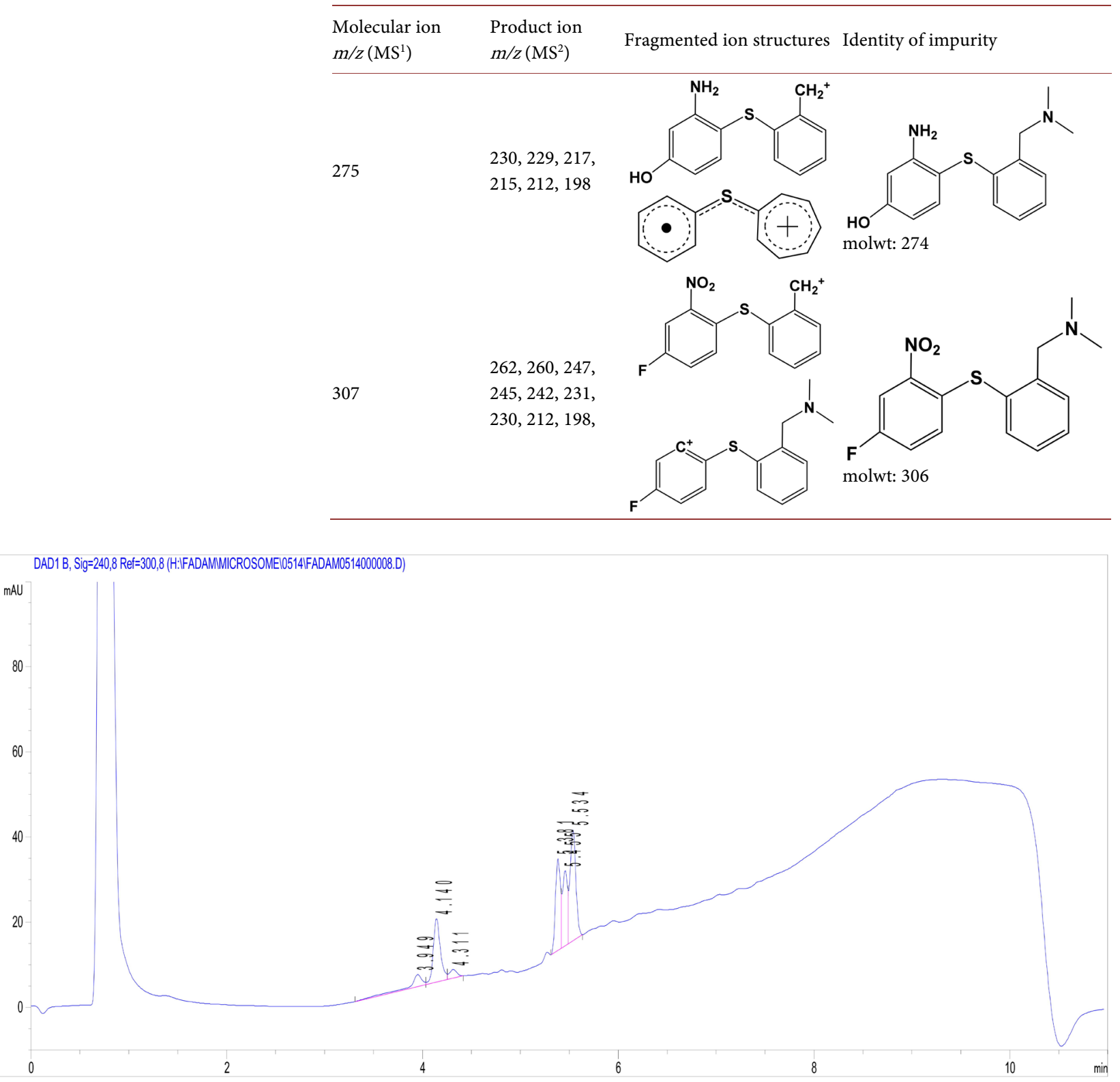

Figure 8. Chromatogram of incubated solution of F-ADAM with liver microsomes.

319 contained a dimethylamine group, and $\mathrm{m} / \mathrm{z}=321$ contained a methoxy group. Furthermore, product ion scanning for every potential metabolite was pursued to acquire the metabolite subunits. The fragmentation spectra for each metabolite are shown in Figures 9-14. According to the collected tandem mass spectra and potential modes of biotransformation [24], the proposed fragment ions and metabolite structures are shown alongside the mass spectra, respectively. For the metabolite with $m / z=293$ (Figure 9) and its fragment ions 248, 246, $\Delta m / z=+16$ compared to F-ADAM $m / z 277$, and fragment ions 232, 230, respectively, the secondary fragment ion $m / z=248 \rightarrow 231\left(-17,-\mathrm{NH}_{3}\right)$ showed that 
it contains phenylamine. The others fragmented ion mass of $m / z 293$ is similar to F-ADAM, therefore the metabolite is supposed an oxidative product of sulfur to sulfoxide $(\mathrm{S}=\mathrm{O})$. Although metabolic oxidization $(+\mathrm{O}, \Delta \mathrm{m} / \mathrm{z}=+16$ compared to the parent molecule) might occur for the transformation of phenylamine $\left(\mathrm{PhNH}_{2}\right)$ into phenylhydroxylamine (PhNH-OH) or benzylic carbon into $\left(\mathrm{CH}_{3}\right)_{2} \mathrm{~N}-\mathrm{Bz}-\mathrm{OH}$, both potential metabolites contain hydroxyl moieties, and fragmented mass spectra ought to show a loss of 18 (for $\mathrm{H}_{2} \mathrm{O}, 293 \rightarrow 275$ ), hence both possible metabolites were eliminated. Figure 10 shows the fragmented ion mass spectra for $m / z=263$, a loss of 14 compared to F-ADAM representing demethylation, $-\mathrm{CH}_{3}$ from dimethylamine. Figure 11 shows the fragmented ion mass spectra for $m / z=264$, which was similar to Figure 10 with an $m / z$ parallel shift of 1 unit. This may represent a hydroxyl $(\mathrm{OH})$ group replacing the amino group on the phenyl moiety. Fragmented ion mass spectra showed that phenol does not release a hydroxyl group from the parent ion as indicated by the lack of a $264 \rightarrow 246$ transition, but rather shows a $233 \rightarrow 215$ transition accompanied by molecular rearrangement. The tandem mass spectrum shown in Figure 12 for a metabolite with $\mathrm{m} / \mathrm{z}=319$ was an acetylation product at the phenylamine moiety. The fragmented ion mass spectrum and proposed structure for $m / z=321$ are shown in Figure 13. These data show an $\mathrm{m} / z=303(-18), 289(-32), 275(-46)$ compared to the parent molecular ion, indicating that it contains $-\mathrm{OH},-\mathrm{OCH}_{3}$ and $-\mathrm{COOH}$ groups. This metabolite underwent oxidative defluorination, eliminating its efficacy as a radiotracer. The metabolite with $\mathrm{m} / \mathrm{z}=334$ showed $\Delta m / z=+57$ compared to F-ADAM, which is believed to result from a glycine conjugated product of phenylamine [24] (Figure 14).

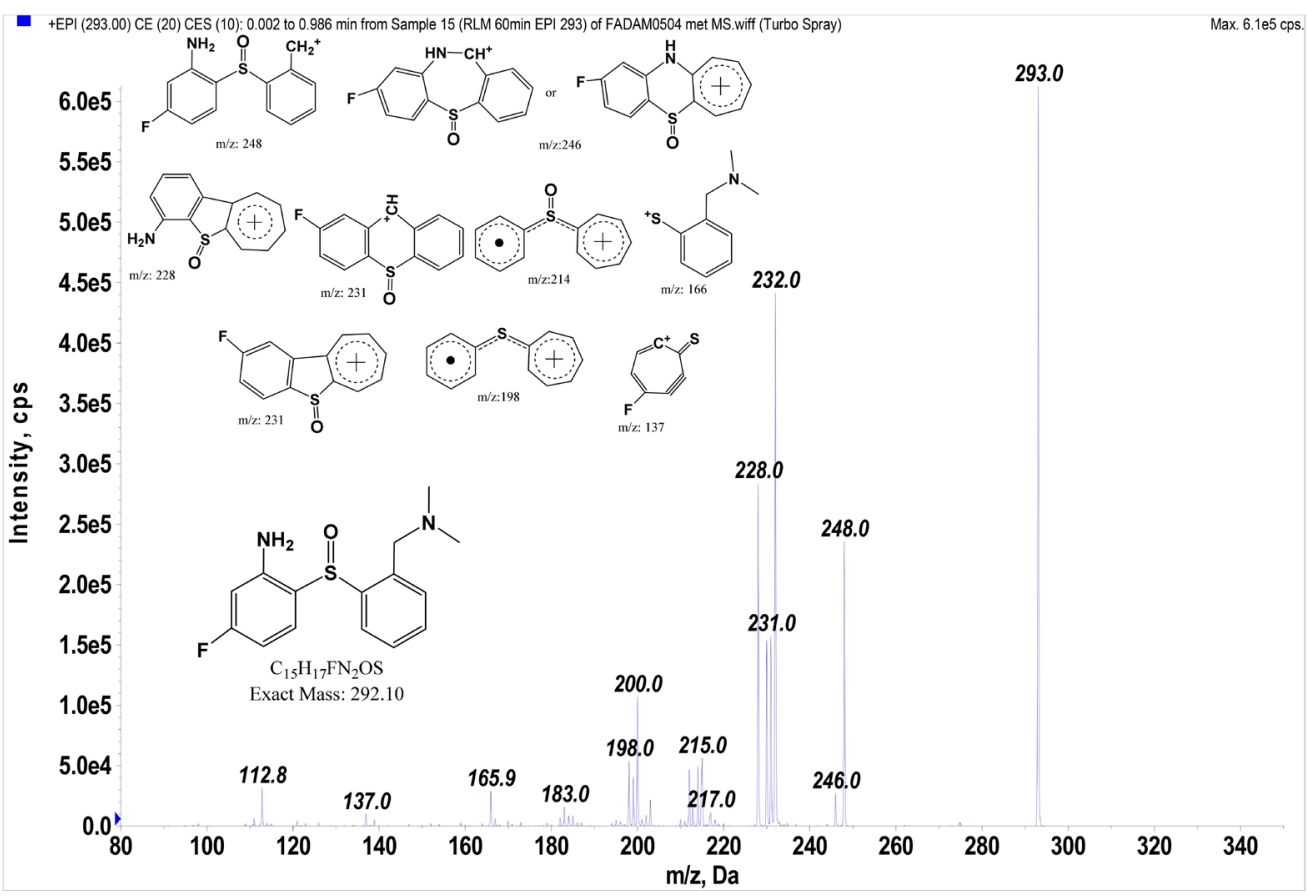

Figure 9. Fragmented ion mass spectra of $m / z=293$ and supposed metabolite identity. 


\section{Conclusion}

In this study of F-ADAM, the identity of unstable derivatives, synthetic impurities, and metabolites by hepatic enzymes were determined using electrospray ionization triple quadrupole tandem mass spectrometry and summarized in Figure 15 and Figure 16, respectively. These data reveal the origin of impurities

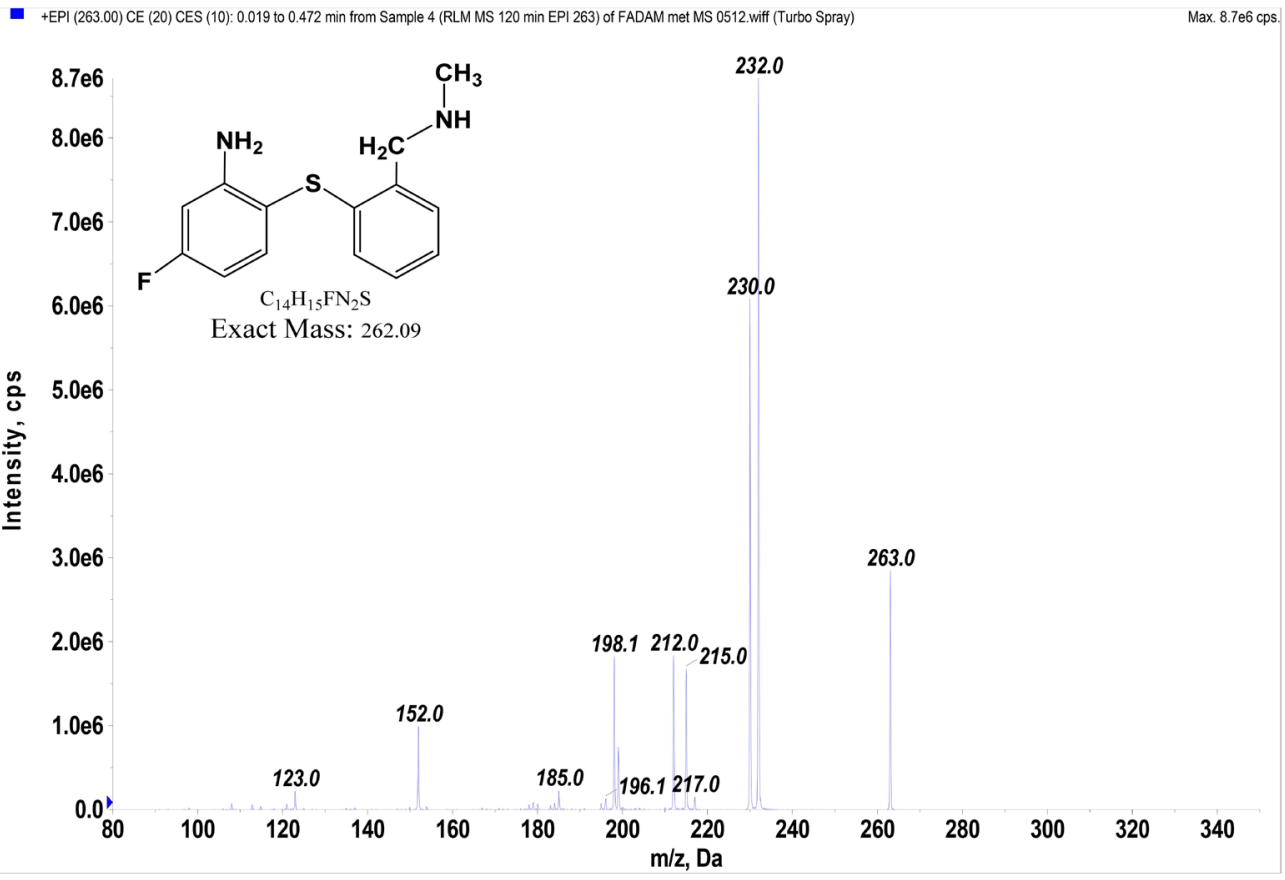

Figure 10. Fragmented ion mass spectra of $m / z=263$ and supposed metabolite identity.

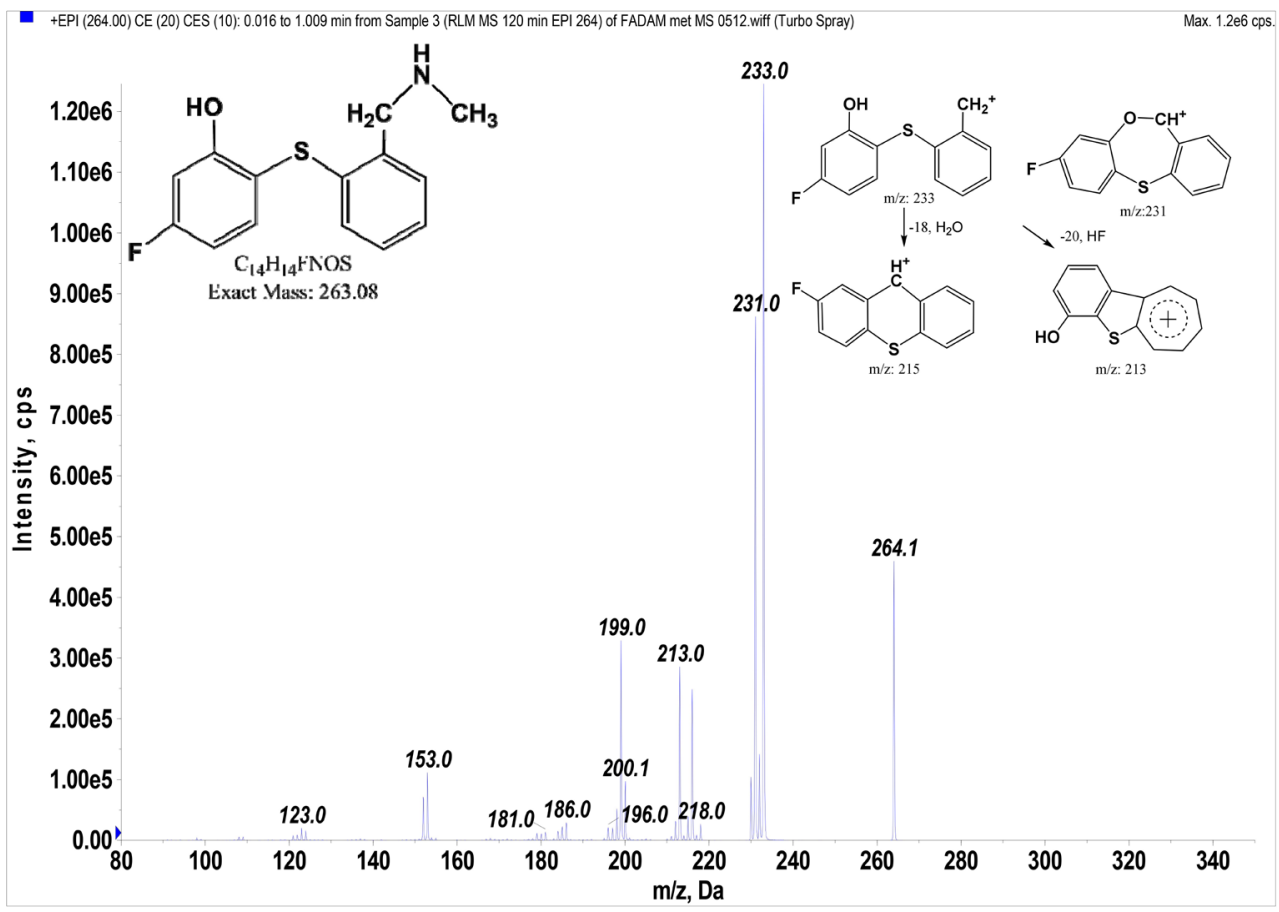

Figure 11. Fragmented ion mass spectra of $m / z=264$ and supposed metabolite identity. 
resulting from the F-ADAM synthetic process, as well as derivatives from its metabolic scheme. The metabolic scheme shows that the biosystem transforms the F-ADAM by increasing the molecular polarity, defluorination, deamination, and blocking the electron pair donating atoms $(\mathrm{N}$ and $\mathrm{S})$ to metalloprotein.

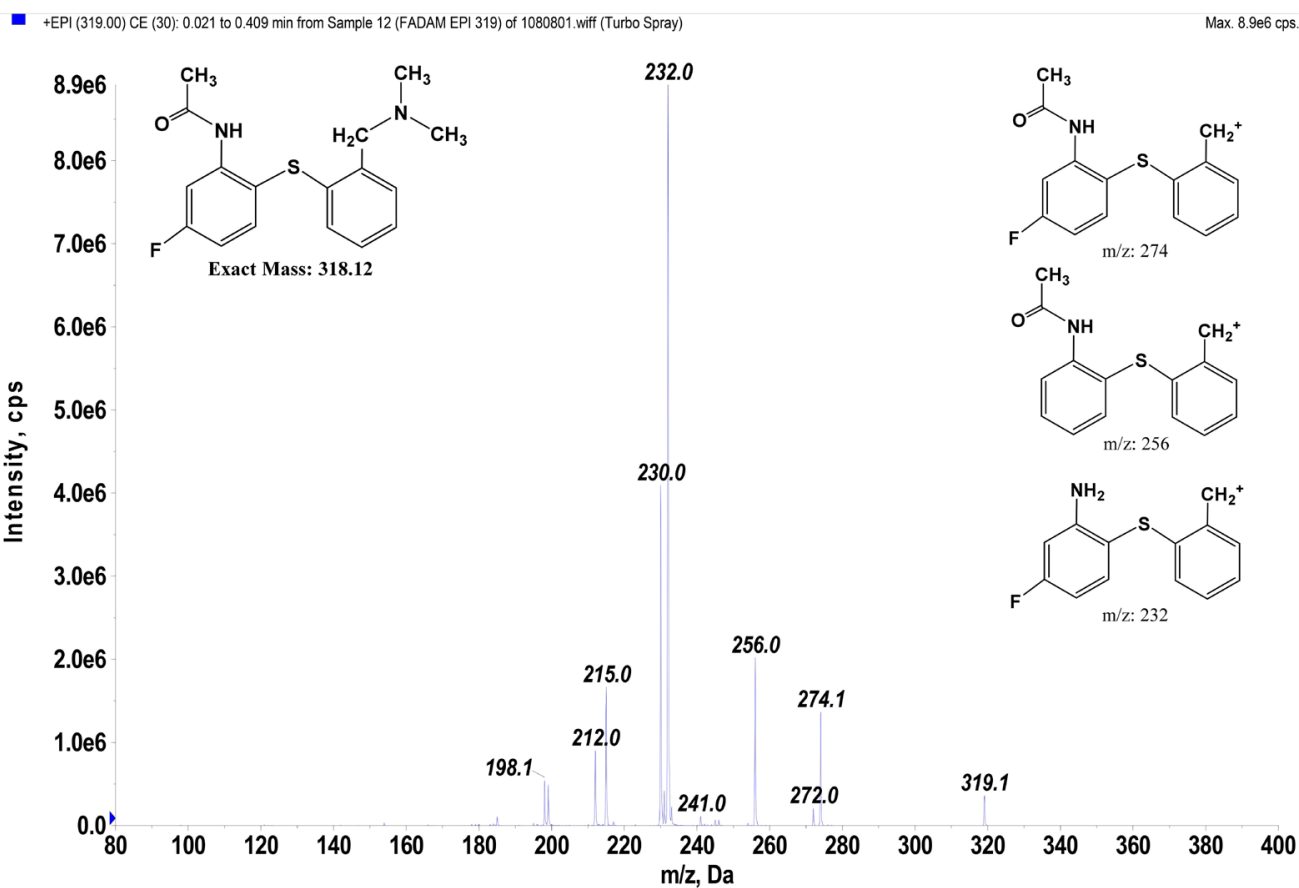

Figure 12. Tandem mass spectra of metabolite $m / z=319$ and proposed structures for metabolite and fragmented ions.

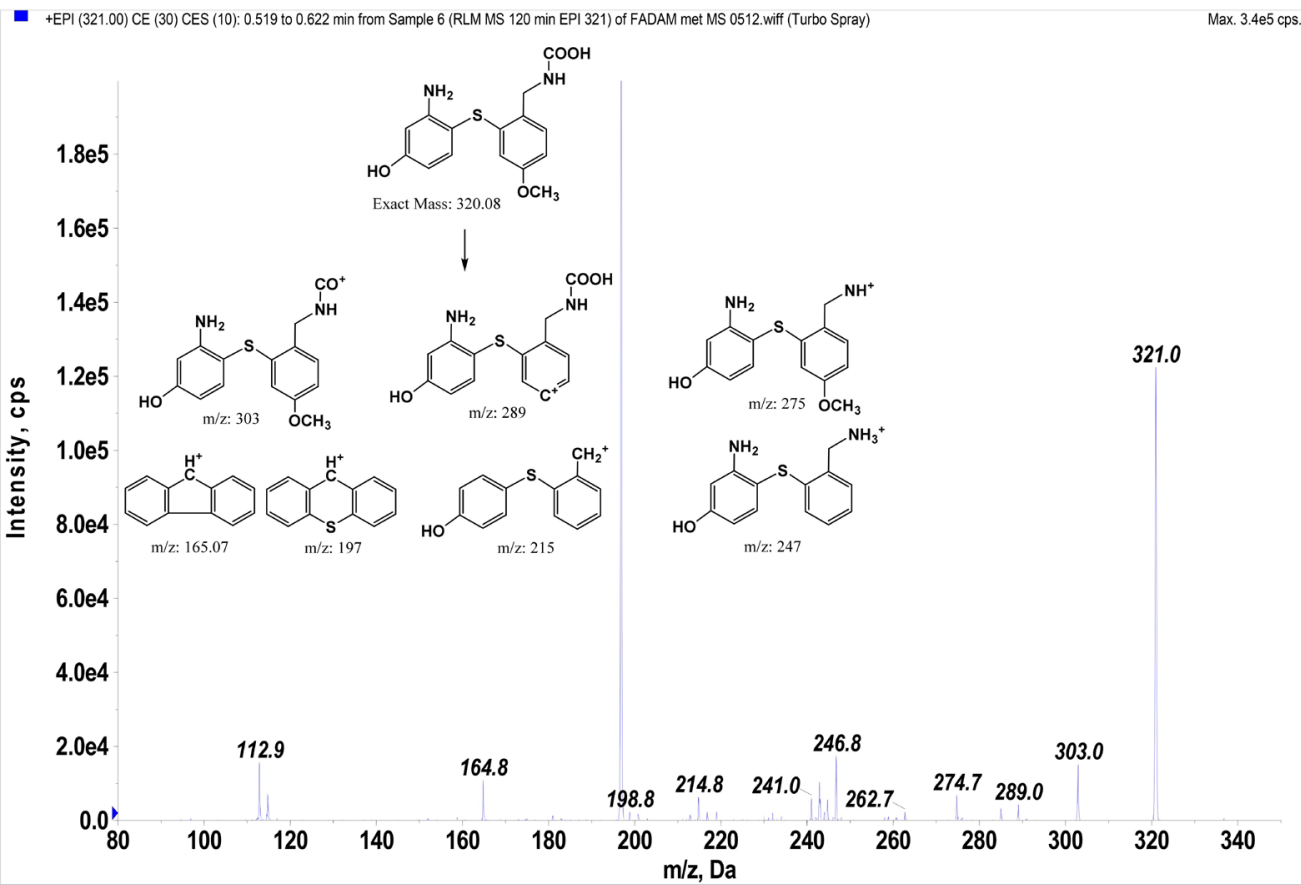

Figure 13. Fragmented ion mass spectra of $m / z=321$ and supposed metabolite and fragmented ions structure. 


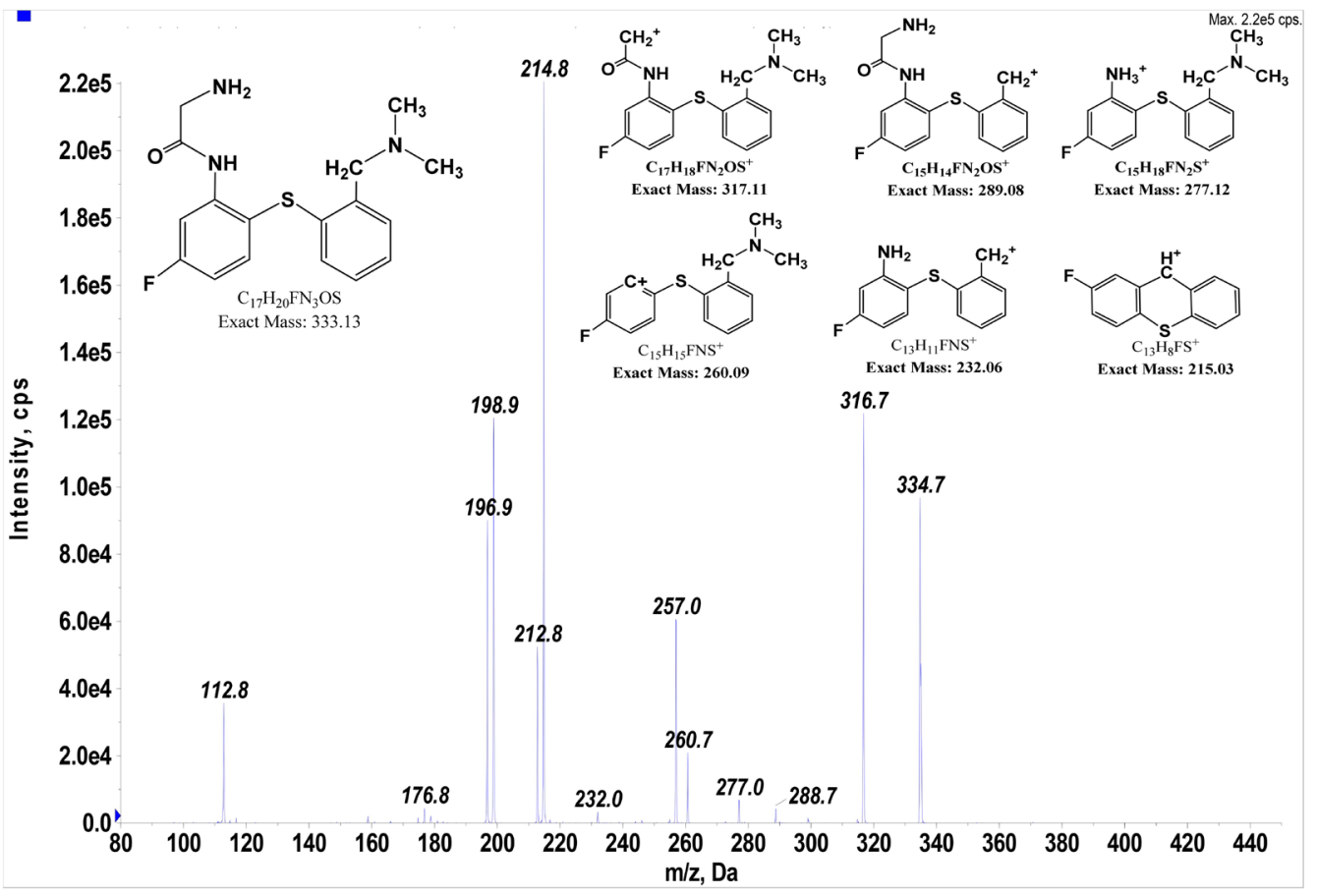

Figure 14. Fragmented ion mass spectra of $\mathrm{m} / \mathrm{z}=334$ and supposed metabolite and fragmented ions structure.

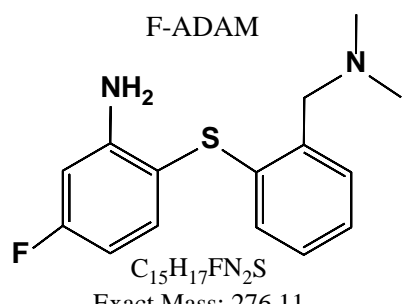<smiles>COc1ccc(Sc2ccccc2CN(C)CC(C)(C)C)c(N)c1</smiles>

dissolved in $\mathrm{CH}_{3} \mathrm{OH}$, unstable, nucleophilic substitution by $\mathrm{CH}_{3} \mathrm{O}^{-}$and $\mathrm{F}^{-}$leaving<smiles>CCOC(=O)N(C)c1c(Sc2ccccc2CN(C)C)ccc(F)c1Cl</smiles>

possible byproduct of starting material

Figure 15. Unstable product while dissolved in methanol and contaminated impurity in synthetic tested standard, F-ADAM. 


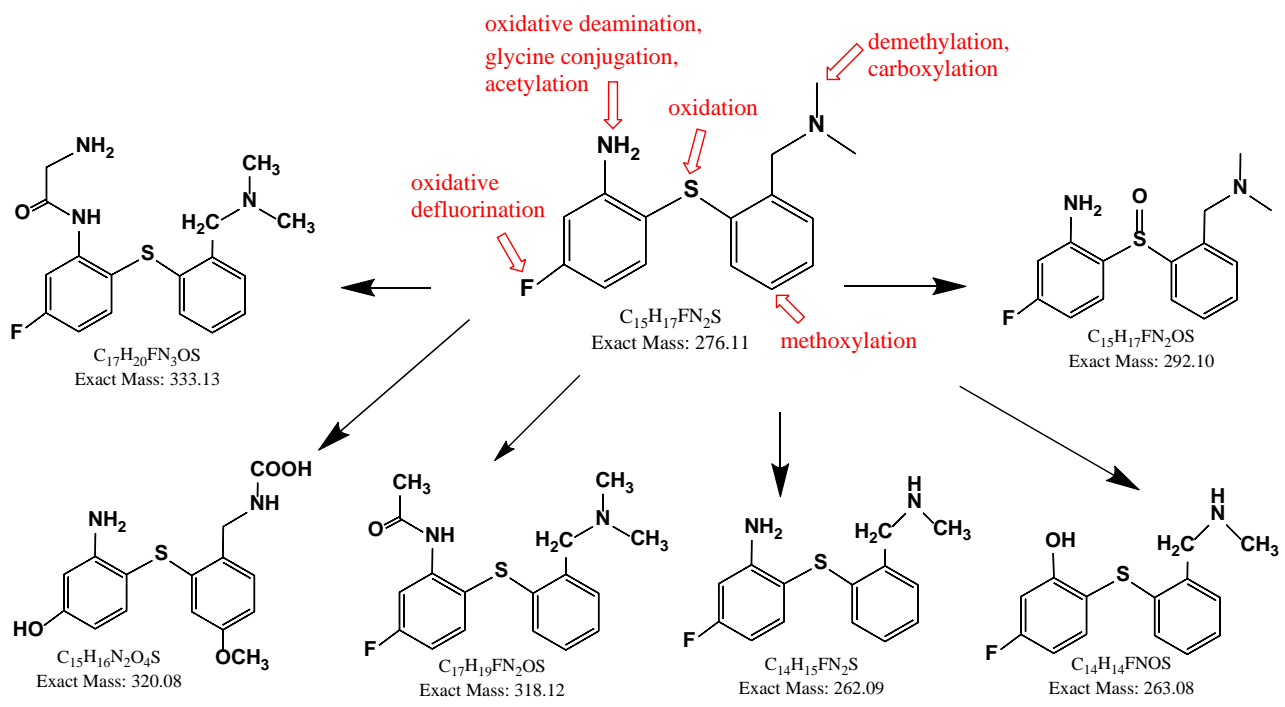

Figure 16. Metabolic scheme of F-ADAM by rat liver microsomes.

Therefore, these metabolites do not bind to the SERT or PET traceable any more, which are decommissioned and eliminated from the body through urine. These findings may facilitate the optimization of the synthetic process and the proper use of F-ADAM as a PET radio-imaging agent for assessment of SERT function.

\section{Acknowledgements}

This research was supported by a grant from the Atomic Energy Council, Taiwan [Grant No. AIE-01030204]. The authors thank Enago group's English editing service.

\section{Conflicts of Interest}

The authors declare no conflicts of interest regarding the publication of this paper.

\section{References}

[1] Kish, S.J. (2002) How Strong Is the Evidence That Brain Serotonin Neurons Are Damaged in Human Users of Ecstasy? Pharmacology Biochemistry and Behavior, 71, 845-855. https://doi.org/10.1016/S0091-3057(01)00708-0

[2] Chiavegatto, S., Dawson, V.L., Mamounas, L.A., et al. (2001) Brain Serotonin Dysfunction Accounts for Aggression in Male Mice Lacking Neuronal Nitric Oxide Synthase. Proceedings of the National Academy of Sciences of the United States of America, 98, 1277-1281. https://doi.org/10.1073/pnas.98.3.1277

[3] Chen, Y.A., Huang, W.S., Lin, Y.S., et al. (2012) Characterization of [ $\left.{ }^{18} \mathrm{~F}\right]-\mathrm{ADAM}$ as an Imaging Agent for SERT in Non-Human Primate Brain Using PET: A Dynamic Study. Nuclear Medicine and Biology, 39, 279-285. https://doi.org/10.1016/j.nucmedbio.2011.08.002

[4] Lin, S.H., Lee, L.T. and Yang, Y.K. (2014) Serotonin and Mental Disorders: A Concise Review on Molecular Neuroimaging Evidence. Clinical Psychopharmacology 
and Neuroscience, 12, 196-202. https://doi.org/10.9758/cpn.2014.12.3.196

[5] Huang, Y.Y., Cheng, C.Y., Huang, W.S., et al. (2014) Toxicity and Radiation Dosimetry Studies of the Serotonin Transporter Radioligand $\left[{ }^{18} \mathrm{~F}\right]$ AFM in Rats and Monkeys. EJNMMI Research, 4, 71-82. https://doi.org/10.1186/s13550-014-0071-1

[6] Sambunaris, A., Hesselink, J.K., Pinder, R., Panagides, J.S. and Stahl, M. (1997) Development of New Antidepressants. The Journal of Clinical Psychiatry, 58, 40-53.

[7] Ma, K.H., Huang, W.S., Kuo, Y.Y., et al. (2009) Validation of 4-[ $\left.{ }^{18} \mathrm{~F}\right]-\mathrm{ADAM}$ as a SERT Imaging Agent Using Micro-PET and Autoradiography. NeuroImage, 45, 687-693. https://doi.org/10.1016/j.neuroimage.2008.12.060

[8] Lundberg, J., Odano, I., Olsson, H., Halldin, C. and Farde, L. (2005) Quantification of ${ }^{11} \mathrm{C}$-MADAM Binding to the Serotonin Transporter in the Human Brain. The Journal of Nuclear Medicine, 46, 1505-1515.

[9] Shiue, G.G., Choi, S.R., Fang, P., Hou, C., Acton, P.D., Cardi, C., et al. (2003) N,N-Dimethyl-2-(2-amino-4- $\left({ }^{18}\right)$ F-fluorophenylthio)benzylamine $\quad\left(4-{ }^{18} \mathrm{~F}-\mathrm{ADAM}\right)$ : An Improved PET Radioligand for Serotonin. The Journal of Nuclear Medicine, 44, 1890-1897. http://jnm.snmjournals.org/content/44/12/1890

[10] Shih, J.H., Ma, K.H., Chen, C.F., et al. (2016) Evaluation of Brain SERT Occupancy by Resveratrol against MDMA-Induced Neurobiological and Behavioral Changes in

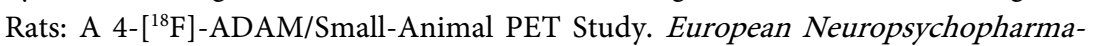
cology, 26, 92-104. https://doi.org/10.1016/j.euroneuro.2015.11.001

[11] Oya, S., Choi, S.R., Hou, C., Mu, M., Kung, M.-P., Acton, P.D., et al. (2000) 2-((2-((Dimethylamino)methyl)phenyl)thio)-5-iodo-phenylamine (ADAM): An Improved Serotonin Transporter Ligand. Nuclear Medicine and Biology, 27, 249-254. https://doi.org/10.1016/S0969-8051(00)00084-6

[12] Lin, K.J., Ye, X.X., Yen, T.C., et al. (2002) Biodistribution Study of [123I]ADAM in Mice: Correlation with Whole Body Autoradiography. Nuclear Medicine and Biology, 29, 643-650. https://doi.org/10.1016/S0969-8051(02)00323-2

[13] Halldin, C., Lundberg, J., Savago, J., et al. (2005) $\left[{ }^{11}\right.$ C]MADAM, a New Serotonin Transporter Radioligand Characterized in the Monkey Brain by PET. Synapse, 58, 173-183. https://doi.org/10.1002/syn.20189

[14] Shiue, G.G., Fang, P. and Shiue, C.Y. (2003) Synthesis of N,N-Dimethyl-2(2-amino-4-[ $\left.{ }^{18} \mathrm{~F}\right]$ fluorophenylthio)benzylamine as a Serotonin Transporter Imaging Agent. Applied Radiation and Isotopes, 58, 183-191. https://doi.org/10.1016/S0969-8043(02)00271-3

[15] Cheng, C.Y., Chou, T.K. and Shiue, C.Y. (2016) Fully Automated One-Pot Two-Step Synthesis of $4-\left[{ }^{18} \mathrm{~F}\right]-A D A M$, a Potent Serotonin Transporter Imaging Agent. Applied Radiation and Isotopes, 110, 8-15.

https://doi.org/10.1016/j.apradiso.2015.12.052

[16] Weng, S.J., Shiue, C.Y., Huang, W.S., et al. (2013) PET Imaging of Serotonin Transporters with $4-\left[{ }^{18} \mathrm{~F}\right]-\mathrm{ADAM}$ in a Parkinsonian Rat Model. Cell Transplantation, 22, 1295-1305. https://doi.org/10.3727/096368912X658683

[17] Chiu, C.H., Li, I.H., Weng, S.J., et al. (2016) PET Imaging of Serotonin Transporters with $4-\left[{ }^{18} \mathrm{~F}\right]-\mathrm{ADAM}$ in a Parkinsonian Rat Model with Porcine Neural Xenografts. Cell Transplantation, 25, 301-311. https://doi.org/10.3727/096368915X688236

[18] Huang, Y.Y., Huang, W.S., Ma, K.H., et al. (2012) Synthesis and Comparison of 4- $\left[{ }^{18} \mathrm{~F}\right] \mathrm{F}-A D A M, 2-\left[{ }^{18} \mathrm{~F}\right] \mathrm{F}-A D A M, N-D e s m e t h y l-4-\left[{ }^{18} \mathrm{~F}\right] \mathrm{F}-A D A M$ and $\left[{ }^{18} \mathrm{~F}\right] \mathrm{F}-\mathrm{AFM}$ as Serotonin Transporter Imaging Agents. Applied Radiation and Isotopes, 70, 2298-2307. https://doi.org/10.1016/j.apradiso.2012.06.005 
[19] Ramanathan, D.M. and LeLacheur, R.M. (2008) Evolving Role of Mass Spectrometry in Drug Discovery and Development. In: Ramanathan, R., Ed., Mass Spectrometry in Drug Metabolism and Pharmacokinetics, Wiley, Hoboken, Ch. 1. https://doi.org/10.1002/9780470409817.ch1

[20] Pilaniya, K., Chandrawanshi, H.K., Pilaniya, U., et al. (2010) Recent Trends in the Impurity Profile of Pharmaceuticals. Journal of Advanced Pharmaceutical Technology \& Research, 1, 302-310. https://doi.org/10.4103/0110-5558.72422

[21] “Mammalian Liver Microsomes Guidelines for Use" Protocol, TF000017 Rev. 2.0, BD Biosciences.

[22] Hill, J.R. (2003) In Vitro Drug Metabolism Using Liver Microsomes. Current Protocols in Pharmacology Unit 7.8.1-7.8.11.

[23] Yang, H.H., Liu, K.T., Hsia, Y.C., et al. (2010) Development and Validation of an HPLC Method for Determination of Purity of Sn-ADAM, a Novel Precursor of Serotonin Transporter SPECT Imaging Agent I-123-ADAM. Journal of Food and Drug Analysis, 18, 307-318. https://doi.org/10.38212/2224-6614.2272

[24] Ramanathan, R., Comezogulu, S.N. and Humphreys, W.G. (2009) Metabolite Identification Strategies and Procedures, Table 5.1. In: Korfmacher, W.A., Ed., Using Mass Spectrometry for Drug Metabolism Studies, 2nd Edition, CRC Press, Boca Raton, Ch. 5. 